\title{
Covering and Piercing Disks with Two Centers
}

\author{
Hee-Kap Ahn* Sang-Sub Kim ${ }^{\dagger} \quad$ Christian Knauer ${ }^{\dagger} \quad$ Lena Schlipf ${ }^{\ddagger}$ \\ Chan-Su Shin ${ }^{\S} \quad$ Antoine Vigneron $₫$
}

\begin{abstract}
We give exact and approximation algorithms for two-center problems when the input is a set $\mathcal{D}$ of disks in the plane. We first study the problem of finding two smallest congruent disks such that each disk in $\mathcal{D}$ intersects one of these two disks. Then we study the problem of covering the set $\mathcal{D}$ by two smallest congruent disks.
\end{abstract}

\section{Introduction}

The standard two-center problem is a well known and extensively studied problem: Given a set $P$ of $n$ points in the plane, find two smallest congruent disks that cover all points in $P$. The best known deterministic algorithm runs in $O\left(n \log ^{2} n \log ^{2} \log n\right)$ [4 and there is a randomized algorithm with expected running time $O\left(n \log ^{2} n\right)[8$. There has also been a fair amount of work on several variations of the two-center problem: for instance, the two-center problem for weighted points [7], and for a convex polygon [17].

In this paper we consider new versions of the problem where the input consists of a set $\mathcal{D}$ of $n$ disks (instead of points): In the intersection problem we want to compute two smallest congruent disks $C_{1}$ and $C_{2}$ such that each disk in $\mathcal{D}$ intersects $C_{1}$ or $C_{2}$, while in the covering problem, all disks in $\mathcal{D}$ have to be contained in the union of $C_{1}$ and $C_{2}$. To the best of our knowledge these problems have not been considered so far. However, linear-time algorithms are known for both the covering and the intersection problem with only one disk [9, 12, 13.

Our results. In order to solve the intersection problem, we first consider the two-piercing problem: Given a set of disks, decide whether there exist two points such that each disk contains at least one of these points. We show that this problem can be solved in $O\left(n^{2} \log ^{2} n\right)$ expected time and $O\left(n^{2} \log ^{2} \log \log n\right)$ deterministic time. Using these algorithms we can solve the intersection problem in $O\left(n^{2} \log ^{3} n\right)$ expected time and $O\left(n^{2} \log ^{4} n \log \log n\right)$ deterministic time.

\footnotetext{
${ }^{*}$ Department of Computer Science and Engineering, POSTECH, Pohang, Korea. \{heekap, helmet1981\}@postech.ac.kr

${ }^{\dagger}$ Institute of Computer Science, Universität Bayreuth, 95440 Bayreuth, Germany. christian.knauer@uni-bayreuth.de

${ }^{\ddagger}$ Institute of Computer Science, Freie Universität Berlin, Germany. schlipf@mi.fu-berlin.de

${ }^{\S}$ Department of Digital and Information Engineering, Hankuk University of Foreign Studies, Yongin, Korea. cssin@hufs.ac.kr

${ }^{\top}$ Geometric Modeling and Scientific Visualization Center, KAUST, Thuwal, Saudi Arabia. antoine.vigneron@kaust.edu.sa
} 
For the covering problem we consider two cases: In the restricted case each $D \in \mathcal{D}$ has to be fully covered by one of the disks $C_{1}$ or $C_{2}$. In the general case a disk $D \in \mathcal{D}$ can be covered by the union of $C_{1}$ and $C_{2}$. We show how the algorithms for the intersection problem can be used to solve the restricted covering case and present an exact algorithm for the general case. We complement these results by giving efficient approximation algorithms for both cases.

All the results presented in this paper are summarized in the following table.

\begin{tabular}{|l|ll|}
\hline & Exact algorithm & $(1+\epsilon)$-approximation \\
\hline Intersection problem & $O\left(n^{2} \log ^{4} n \log \log n\right)$ & - \\
& $O\left(n^{2} \log ^{3} n\right)$ expected time & \\
\hline General covering problem & $O\left(n^{3} \log ^{4} n\right)$ & $O\left(n+1 / \epsilon^{3}\right)$ \\
\hline Restricted covering problem & $\begin{array}{l}O\left(n^{2} \log ^{4} n \log \log n\right) \\
\\
\end{array}\left(^{2} \log ^{3} n\right)$ expected time & \\
\hline
\end{tabular}

Notation. The radius of a disk $D$ is denoted by $r(D)$ and its center by $c(D)$. The circle that forms the boundary of $D$ is denoted by $\partial D$.

Without loss of generality, we assume that no disk in $\mathcal{D}$ contains another disk in $\mathcal{D}$.

\section{Intersecting Disks with Two Centers}

In this section we consider the following intersection problem: Given a set of disks $\mathcal{D}=$ $\left\{D_{1}, \ldots, D_{n}\right\}$, we want to find two smallest congruent disks $C_{1}$ and $C_{2}$ such that each disk $D \in \mathcal{D}$ has a nonempty intersection with $C_{1}$ or $C_{2}$.

Based on the observation below, there is an $O\left(n^{3}\right)$ algorithm for this problem.

Observation 1. Let $\left(C_{1}, C_{2}\right)$ be a pair of optimal covering disks. Let $\ell$ be the bisector of the segment connecting the centers of $C_{1}$ and $C_{2}$. Then, $C_{i} \cap D \neq \emptyset$ for every $D \in \mathcal{D}$ whose center lies on the same side of $\ell$ as the center of $C_{i}$, for $i=\{1,2\}$.

A simple approach would be, for every bipartition of the centers of the disks in $\mathcal{D}$ by a line $\ell$, to compute the smallest disk intersecting the disks on each side of $\ell$, and return the best result over all bipartitions. Since there are $O\left(n^{2}\right)$ such partitions, and the smallest disk intersecting a set of disks can be found in linear time [12, this algorithm runs in $O\left(n^{3}\right)$ time.

We will present faster algorithms for the intersection problem. We first introduce a related problem. For a real number $\delta \geq 0$ and a disk $D$, the $\delta$-inflated disk $D(\delta)$ is a disk concentric to $D$ and whose radius is $r(D)+\delta$. Consider the following decision problem:

Given a value $\delta \geq 0$, are there two points $p_{1}$ and $p_{2}$ such that $D(\delta) \cap\left\{p_{1}, p_{2}\right\} \neq \emptyset$ for every $D \in \mathcal{D}$ ?

This problem is related to our original problem in the following way. The above condition holds with $\delta$ if and only if the two disks centered at $p_{1}$ and $p_{2}$ with radius $\delta$ intersect all disks $D \in \mathcal{D}$. Therefore the two disks centered at $p_{1}$ and $p_{2}$ with radius $\delta^{*}$ are a solution to the intersection problem, where $\delta^{*}$ is the minimum value for which the answer to the decision problem is "yes". 


\subsection{Decision Algorithm}

Given a value $\delta \geq 0$, we construct the arrangement of the $\delta$-inflated disks $D_{i}(\delta), i=1 \ldots n$ in the plane. This arrangement consists of $O\left(n^{2}\right)$ cells, each cell being a 0,1 , or 2 -face. We traverse all the cells in the arrangement in a depth-first manner and do the followings: We place one center point, say $p_{1}$, in a cell. The algorithm returns "yes" if all the disks that do not contain $p_{1}$ have a nonempty common intersection. Otherwise, we move $p_{1}$ to a neighboring cell, and repeat the test until we visit every cell. This naïve approach leads to a running time $O\left(n^{3}\right)$ : we traverse $O\left(n^{2}\right)$ cells, and each cell can be handled in linear time.

The following approach allows us to improve this running time by almost a linear factor. We consider a traversal of the arrangement of the $\delta$-inflated disks by a path $\gamma$ that crosses only $O\left(n^{2}\right)$ cells, that is, some cells may be crossed several times, but on average each cell is crossed $O(1)$ times. It can be achieved by choosing the path $\gamma$ to be the Eulerian tour of the depth-first search tree from the naïve approach.

While we move the center $p_{1}$ along $\gamma$ and traverse the arrangement, we want to know whether the set of disks $\mathcal{D}^{\prime}$ that do not contain $p_{1}$ have a non-empty intersection. To do this efficiently, we use a segment tree [6]. Each disk of $\mathcal{D}$ may appear or disappear several times during the traversal of $\gamma$ : each time we cross the boundary of a cell, one disk is inserted or deleted from $\mathcal{D}^{\prime}$. So each disk appears in $\mathcal{D}^{\prime}$ along one or several segments of $\gamma$. We store these segments in a segment tree. As there are only $O\left(n^{2}\right)$ crossings with cell boundaries along $\gamma$, this segment tree is built over a total of $O\left(n^{2}\right)$ endpoints and thus has total size $O\left(n^{2} \log n\right)$ : Each segment of $\gamma$ along which a given disk of $\mathcal{D}$ is in $\mathcal{D}^{\prime}$ is inserted in $O(\log n)$ nodes of the segment tree. Each node $v$ of the segment tree stores a set $\mathcal{D}_{v} \subseteq \mathcal{D}$ of input disks; from the discussion above, they represent disks that do not contain $p_{1}$ during the whole segment of $\gamma$ that is represented by $v$. In addition, we store at node $v$ the intersection $I_{v}=\bigcap \mathcal{D}_{v}$ of the disks stored at $v$. Each such intersection $I_{v}$ is a convex set bounded by $O(n)$ circular $\operatorname{arcs}$, so we store them as an array of circular arcs sorted along the boundary of $I_{v}$. In total it takes $O\left(n^{2} \log ^{2} n\right)$ time to compute the intersections $I_{v}$ for all nodes $v$ in the segment tree, since each disk is stored at $O(n \log n)$ nodes on average and the intersection of $k$ disks can be computed in $O(k \log k)$ time.

We now need to decide whether at some point, when $p_{1}$ moves along $\gamma$, the intersection of the disks in $\mathcal{D}^{\prime}$ (that is, disks that do not contain $p_{1}$ ) is nonempty. To do this, we consider each leaf of the segment tree separately. At each leaf, we test whether the intersection of the disks stored at this leaf and all its ancestors is non-empty. So it reduces to emptiness testing for a collection of $O(\log n)$ circular polygons with $O(n)$ circular arcs each. We can solve this in $O\left(\log ^{2} n\right)$ expected time by randomized convex programming [5, 16], using $O(\log n)$ of the following primitive operations:

1. Given $I_{i}, I_{j}$ and vector $a \in \mathbb{R}^{2}$, find the extreme point $v \in I_{i} \cap I_{j}$ that minimizes $a \cdot v$.

2. Given $I_{i}$ and a point $p$, decide whether $p \in I_{i}$.

We can also solve this problem in $O\left(\log ^{2} n \log \log n\right)$ time using deterministic convex programming [3. So we obtain the following result:

Lemma 2. Given a value $\delta \geq 0$, we can decide in $O\left(n^{2} \log ^{2} n\right)$ expected time or in $O\left(n^{2} \log ^{2} n \log \log n\right)$ worst-case time whether there exist two points such that every $\delta$-inflated disk intersects at least one of them. 


\subsection{Optimization Algorithm}

The following lemma shows that the optimum $\delta^{*}$ can be found in a set of $O\left(n^{3}\right)$ possible values.

Lemma 3. When $\delta=\delta^{*}, p_{1}$ or $p_{2}$ is a common boundary point of three $\delta^{*}$-inflated disks, a tangent point of two $\delta^{*}$-inflated disks or $\delta^{*}=0$.

Proof. Suppose that this is not the case. Then the common intersection of the disks containing $p_{1}$ has nonempty interior. Similarly, the common intersection of the disks containing $p_{2}$ has nonempty interior. Let $p_{1}^{\prime}$ and $p_{2}^{\prime}$ be points in the interiors, one from each common intersection. Then there is a value $\delta^{\prime}<\delta$ satisfying $D\left(\delta^{\prime}\right) \cap\left\{p_{1}^{\prime}, p_{2}^{\prime}\right\} \neq \emptyset$ for every $D \in \mathcal{D}$. But we also assumed that $\delta^{*} \neq 0$.

\section{Finding $\delta^{*}$}

Due to Lemma 3 we consider only discrete values of $\delta$ for which one of the events defined in Lemma 3 occurs. Whether $\delta^{*}=0$ can be tested with the decision algorithm in $O\left(n^{2} \log ^{2} n\right)$ expected time or in $O\left(n^{2} \log ^{2} n \log \log n\right)$ worst-case time. So from now on, we assume that $p_{1}$ or $p_{2}$ is a common boundary point of three $\delta$-inflated disks or a tangent point of two $\delta$-inflated disks.

In order to compute all possible values for $\delta$, we construct a frustum $f_{i} \in \mathbb{R}^{3}$ for each disk $D_{i} \in \mathcal{D}$. The bottom base of the frustum $f_{i}$ is $D_{i}$ lying in the plane $z=0$. The intersection of $f_{i}$ and the plane $z=\delta$ is $D_{i}(\delta)$. The top base of $f_{i}$ is $D_{i}\left(\delta_{\max }\right)$, where $\delta_{\max }$ is the minimum radius of the disk intersecting all disks in $\mathcal{D}$. Clearly, the optimal value of $\delta$ is in $\left[0, \delta_{\max }\right]$.

\subsubsection{Event points and their corresponding radii.}

Consider the case that $p_{1}=(x, y)$ is the common boundary point of the disks $D_{i}(\delta), D_{j}(\delta)$, and $D_{k}(\delta)$ in the plane. Then the point $p^{\prime}=(x, y, \delta)$ is the common boundary point of three frustums $f_{i}, f_{j}$, and $f_{k}$. Consider now the case that $p_{1}=(x, y)$ is the tangent point of $D_{i}(\delta)$ and $D_{j}(\delta)$. Then the point $p^{\prime}=(x, y, \delta)$ is the point with the smallest $z$-value on the intersection curve of $f_{i}$ and $f_{j}$. We call such a point the tangent point of two frustums. Hence, in order to find the points $p_{1}$ and $p_{2}$, all the tangent points and the common boundary points of the frustums have to be considered. There are $O\left(n^{2}\right)$ tangent points and $O\left(n^{3}\right)$ common boundary points, therefore there are $O\left(n^{3}\right)$ candidates for the point $p_{1}$ in total (note that for each candidate for $p_{1}$, the corresponding value for $\delta$ is obtained, namely the height of $\left.p_{1}^{\prime}\right)$. Thus, a naïve way to find the minimum value $\delta$ such that there exists two points $p_{1}, p_{2}$ that fulfil the conditions, is to test all candidate $\delta$ values. For each possible $\delta$ value, we can determine if there are two points $p_{1}, p_{2}$ such that all $D(\delta)$ are intersected by $p_{2}$ or $p_{1}$ (as argued above). The solution is the smallest value $\delta^{*}$ at which the decision algorithm in Section 2.1 returns "yes". This leads to a running time of $O\left(n^{5} \log ^{2} n\right)$ expected time or $O\left(n^{5} \log ^{2} n \log \log n\right)$ deterministic time.

In order to improve the running time we use an implicit binary search.

\subsubsection{Implicit binary search.}

We perform an implicit binary search on the $\delta$ values corresponding to these common boundary points. As argued above, $p_{1}$ is the projection of a point $p^{\prime}$ which is a tangent point of two 
frustums or a common boundary point of three frustums, i.e., a vertex of the arrangement $\mathcal{A}$ of the $n$ frustums $f_{1}, \ldots f_{n}$; the complexity of $\mathcal{A}$ is $O\left(n^{3}\right)$. We now describe how to perform the binary search over the vertices of $\mathcal{A}$ in an implicit way:

Binary search on a coarse list of events. We first consider $O\left(n^{2}\right)$ pairs of frustums and compute the tangent point of each pair. Then we randomly select $O\left(n^{2} \log n\right)$ triples of frustums and compute the common boundary point of each triple. Since $\delta^{*} \in\left[0, \delta_{\max }\right]$, we only consider points whose $z$-value is in this interval. Clearly, these points are vertices of $\mathcal{A}$ and hence we randomly select $O\left(n^{2} \log n\right)$ vertices from $\mathcal{A}$. We sort their radii associated with them in $O\left(n^{2} \log ^{2} n\right)$ time. By a binary search with the decision algorithm in Section 2.1. we determine two consecutive radii $\delta_{i}$ and $\delta_{i+1}$ such that $\delta^{*}$ is between $\delta_{i}$ and $\delta_{i+1}$. This takes $O\left(n^{2} \log ^{3} n\right)$ time. Since the vertices were picked randomly, the strip $W\left[\delta_{i}, \delta_{i+1}\right]$ bounded by the two planes $z:=\delta_{i}$ and $z:=\delta_{i+1}$ contains only $k=O(n)$ vertices of $\mathcal{A}$ with high probability [14, Section 5].

Zooming into the interval. We compute all the $k$ vertices in $W\left[\delta_{i}, \delta_{i+1}\right]$ by a standard sweep-plane algorithm in $O\left(k \log n+n^{2} \log n\right)$ time as follows: First, we compute the intersection of the sweeping plane at $z:=\delta_{i}$ with the frustums $f_{1}, \ldots, f_{n}$. This intersection forms a two-dimensional arrangement of $O(n)$ circles with $O\left(n^{2}\right)$ total complexity, and we can compute it in $O\left(n^{2} \log n\right)$ time. We next construct the portion of the arrangement $\mathcal{A}$ in $W\left[\delta_{i}, \delta_{i+1}\right]$ incrementally by sweeping a plane orthogonal to $z$-axis from the intersection at $z:=\delta_{i}$ towards $z:=\delta_{i+1}$. As a result, we can compute the $k=O(n)$ vertices (and the corresponding $O(n)$ radii) in $W\left[\delta_{i}, \delta_{i+1}\right]$ in $O(n \log n)$ time. We abort the sweep if the number $k$ of vertices inside the strip becomes too large and restart the algorithm with a new random sample. This happens only with small probability. In order to find the minimum value $\delta^{*}$, we perform a binary search on these $O(n)$ radii we just computed, using the decision algorithm in Lemma 2. This takes $O\left(n^{2} \log ^{3} n\right)$ expected time. The solution pair of points $p_{1}$ and $p_{2}$ can also be found by the decision algorithm.

To get a deterministic algorithm, we use the parametric search technique, with the deterministic decision algorithm of Lemma 2. As the generic algorithm, we use an algorithm that computes in $O(\log n)$ time the arrangement of the inflated disks using $O\left(n^{2}\right)$ processors [2], so we need to run the decision algorithm $O\left(\log ^{2} n\right)$ times, and the total running time becomes $O\left(n^{2} \log ^{4} n \log \log n\right)$.

Theorem 4. Given a set $\mathcal{D}$ of $n$ disks in the plane, we can compute two smallest congruent disks whose union intersects every disk in $\mathcal{D}$ in $O\left(n^{2} \log ^{3} n\right)$ expected time, and in $O\left(n^{2} \log ^{4} n \log \log n\right)$ deterministic time.

\section{Covering Disks with Two Centers}

In this section we consider the following covering problem: Given a set of disks $\mathcal{D}=\left\{D_{1}, \ldots, D_{n}\right\}$, compute two smallest congruent disks $C_{1}$ and $C_{2}$ such that each disk $D \in \mathcal{D}$ is covered by $C_{1}$ or $C_{2}$. In the general case, a disk $D \in \mathcal{D}$ must be covered by $C_{1} \cup C_{2}$. In the restricted case, each disk $D \in \mathcal{D}$ has to be fully covered by $C_{1}$ or by $C_{2}$. 


\subsection{The General Case}

We first give a characterization of the optimal covering. The optimal covering of a set $\mathcal{D}^{\prime}$ of disks by one disk is determined by at most three disks of $\mathcal{D}^{\prime}$ touching the optimal covering disk such that the convex hull of the contact points contains the center of the covering disk. (See Figure 1(a).)

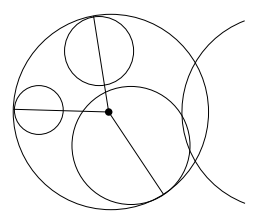

(a)

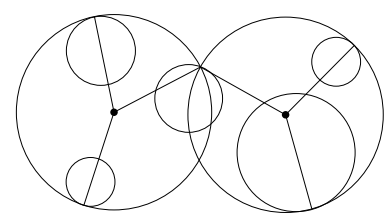

(b)

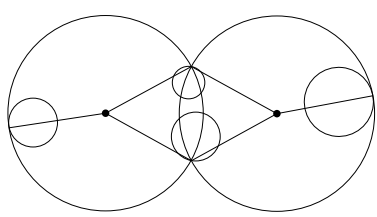

(c)

Figure 1: The three configurations for the optimal 2-center covering of disks.

When covering by two disks, a similar argument applies, and thus the optimal covering disks $\left(C_{1}^{*}, C_{2}^{*}\right)$ are determined by at most five input disks.

Lemma 5. The optimal covering by two disks $C_{1}^{*}, C_{2}^{*}$ satisfies one of the following conditions.

1. For some $i \in\{1,2\}$, the disk $C_{i}^{*}$ is the optimal one-covering of the disks contained in $C_{i}^{*}$, as in Figure 1(a).

2. There is an input disk that is neither fully contained in $C_{1}^{*}$ nor in $C_{2}^{*}$, but contains one point of $\partial C_{1}^{*} \cap \partial C_{2}^{*}$ in its boundary as in Figure $1(b)$.

3. There are two input disks $D_{i}, D_{j}$, possible $i=j$, none of them being fully covered by $C_{1}^{*}$ or $C_{2}^{*}$, such that $D_{i}$ contains one point of $\partial C_{1}^{*} \cap \partial C_{2}^{*}$ and $D_{j}$ contains the other point of $\partial C_{1}^{*} \cap \partial C_{2}^{*}$ in their boundaries as in Figure $1(c)$.

In all cases, each covering disk $C^{*}$ is determined by at most three disks whose contact points contain the center $c\left(C^{*}\right)$ in their convex hull.

Proof. The optimal solution is a pair of congruent disks that achieves a local minimum in radius, that is, we cannot reduce the radius of the covering disks by translating them locally. If one covering disk is completely determined by the input disks contained in it, then this belongs to case 1 . Otherwise, there always exists at least one input disk $D$ such that $D$ is not contained in $C_{i}^{*}$ for all $i \in\{1,2\}$. Moreover such input disks always touch $C_{1}^{*} \cup C_{2}^{*}$ from inside at the intersection points of $\partial C_{1}^{*}$ and $\partial C_{2}^{*}$, otherwise we can always get a pair of smaller congruent covering disks. If only one point of $\partial C_{1}^{*} \cap \partial C_{2}^{*}$ is touched by an input disk $D$, both covering disks are determined by at most two additional disks touching from inside together with $D$ because the covering disks are congruent. If both intersection points of $\partial C_{1}^{*} \cap \partial C_{2}^{*}$ are touched by input disks $D_{i}$ and $D_{j}$, possible $i=j$, one covering disk is determined by one additional disk and the other covering disk by at most one additional disk touching from inside together with $D_{i}$ and $D_{j}$ because the covering disks are congruent. It is not difficult to see that there are two or three touching points of each covering disk that make radial angles at most $\pi$; otherwise we can get a pair of smaller congruent covering disks. 
Using a decision algorithm and the parametric search technique, we can construct an exact algorithm for the general covering problem.

Let $r^{*}$ be the radius of an optimal solution for the general case of covering by two disks. We describe a decision algorithm based on the following lemma that, for a given $r>0$, returns "yes" if $r \geq r^{*}$, and "no" otherwise. (See also Figure 2).

Lemma 6. Assume that $r \geq r^{*}$. Then there exists a pair of congruent disks $C_{1}, C_{2}$ of radius $r$ such that their union contains the input disks, an input disk $D$ touches $C_{1}$ from inside, and one of the following property holds.

(a) $C_{1}$ is identical to $D$.

(b) There is another input disk touching $C_{1}$ from inside.

(c) There is another input disk $D^{\prime}$ such that $D^{\prime}$ is not contained in $C_{2}$, but it touches a common intersection $t$ of $\partial C_{1}$ and $\partial C_{2}$ that is at distance $2 r$ from the touching point of $D$. If this is the case, we say that $D$ and $t$ are aligned with respect to $C_{1}$.

(d) There are two disks $D_{i}$ and $D_{j}$, possibly $i=j$, such that $D_{i}$ touches a common intersection of $\partial C_{1}$ and $\partial C_{2}$, and $D_{j}$ touches the other common intersection of $\partial C_{1}$ and $\partial C_{2}$.

Proof. Let $c_{1}^{*}$ and $c_{2}^{*}$ be the centers of the optimal solution. Imagine that we place two disks at $c_{1}^{*}$ and $c_{2}^{*}$ with radius larger than $r^{*}$, respectively. If $C_{1}$ is already identical to an input disk $D$, it belongs to case (a). Otherwise we translate $C_{1}$ towards $C_{2}$ until it hits an input disk $D$. Then we rotate $C_{1}$ around $D$ in clockwise orientation maintaining $D$ touching $C_{1}$ from inside until the union of $C_{1}$ and $C_{2}$ stops covering the input. If this event is caused by another disk touching $C_{1}$ from inside, it belongs to case (b). Otherwise the event is caused by another disk $D_{i}$ that is hit by one of two common intersections of $\partial C_{1}$ and $\partial C_{2}$ at $t$. If $D$ and $t$ are aligned with respected to $C_{1}$, it belongs to case (c).

Otherwise, we rotate $C_{2}$ around $t$ in counterclockwise until the union of $C_{1}$ and $C_{2}$ stops covering the input. If this event is caused by another disk $D_{j}$ that is hit by the common intersection of $\partial C_{1}$ and $\partial C_{2}$, other than $t$, then it belongs to case (d). Otherwise the event is caused by another disk $D^{\prime}$ touching $C_{2}$ from inside. Thus, $D$ touches $C_{1}$ from inside, $D^{\prime}$ touches $C_{2}$ from inside, and $D_{i}$ touches the common intersection $t$ of $\partial C_{1}$ and $\partial C_{2}$. Imagine that we rotate $C_{1}$ slightly further around $D$ in clockwise. We also rotate $C_{2}$ around $D^{\prime}$ simultaneously such that $D_{i}$ and the rotated copies keep maintaining a common intersection along their boundaries during the rotation. Let $t$ denote the common intersection. We rotate $C_{1}$ and $C_{2}$ in such a way until we encounter an event (1) that another disk $D_{j}$ touches $C_{1}$ or $C_{2}$, (2) that $D_{j}$ touches the other common intersection of $\partial C_{1}$ and $\partial C_{2}$, or (3) that $D$ and $t$ are aligned with respect to $C_{1}$ or $D^{\prime}$ and $t$ are aligned with respect to $C_{2}$. Note that if the event is of type (3), then $D_{i}$ is not contained in the disk centered at $c(D)$ with radius $2 r-r(D)$ or is not contained in the disk centered at $c\left(D^{\prime}\right)$ with radius $2 r-r\left(D^{\prime}\right)$ as in Figure 2 .

\subsubsection{Decision Algorithm.}

The cases are enumerated as in Lemma 6 , Case (a). Choose an input disk $D$. $C_{1}$ has radius $r$ and covers only $D$. Then $C_{2}$ is the smallest disk containing $\mathcal{D} \backslash D$. If the radius of $C_{2}$ is $\leq r$, we return "yes". 


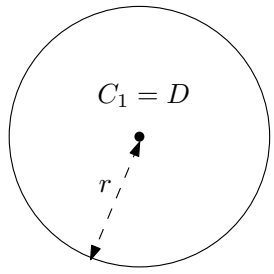

(a)

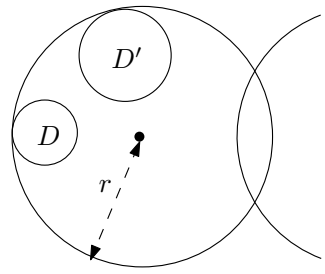

(b)

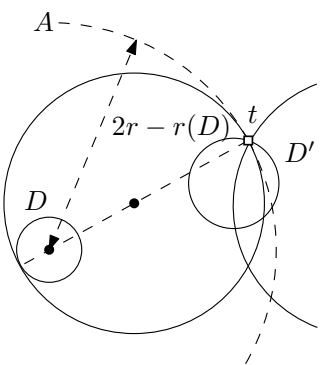

(c)

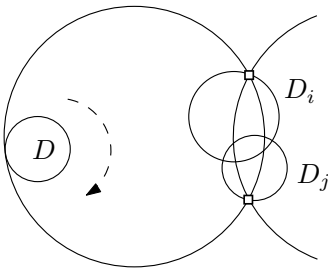

(d)

Figure 2: Four cases for $r \geq r^{*}$.

Case (b). We simply choose a pair of input disks $D$ and $D^{\prime}$. There are two candidates for $C_{1}$, as $C_{1}$ has radius $r$ and touches $D$ and $D^{\prime}$. So we consider separately each of the two candidates for $C_{1}$. Then $C_{2}$ is chosen to be the smallest disk containing the input disks, or the portions of input disks (crescents) that are not covered by $C_{1}$, which can be done in $O(n)$ time. If for one of the two choices of $C_{1}$, the corresponding disk $C_{2}$ has radius $\leq r$, we return "yes".

Case (c). For each input disk $D$, we do the following.

1. For the circle $A$ with center $c(D)$ and radius $2 r-r(D)$, compute $A \cap D^{\prime}$ for every other disk $D^{\prime}$. Let $t$ be such an intersection point.

2. For each $t$,

(a) remove (part of) the input disks covered by the covering disk determined by $D$ and $t$, and compute the smallest disk covering the remaining input.

(b) If this algorithm returns a covering disk with radius $\leq r$, return "yes".

Case (d). For each input disk $D$ that touches $C_{1}$ from inside, we do the following. Let $i$ be the index of the first input disk that the circular arc of $C_{1}$ from the touching point hits in clockwise orientation. Let $j$ be the index of the last input disk that the circular arc leaves. We claim that the number of pairs of type $(i, j)$ is $O(n)$.

This claim can be easily proved by observing that, while we rotate $C_{1}$ around an input disk $D$ in clockwise orientation, $C_{1}$ sweeps the plane and the input disks in such a manner that the first input disk intersected by the arc of $C_{1}$ from the tangent point in clockwise orientation changes only $O(n)$ times; To see this, consider the union of the input disks, which consists of $O(n)$ circular arcs. The last input disk intersected also changes $O(n)$ times. So the pairing along the rotation can be done by scanning two lists (first and last) of disks. For each pair $(i, j)$, we still have some freedom of rotating $C_{1}$ around $D$ within some interval $\left(C_{2}\right.$ changes accordingly.) During the rotation, an input disk not covered by the union of $C_{1}$ and $C_{2}$ may become fully covered by the union, or vice versa. We call such an event an $I / O$ event. Note that an I/O event occurs only when an input disk touches $C_{1}$ or $C_{2}$ from inside. Again, we claim that the number of $\mathrm{I} / \mathrm{O}$ events for each pair $(i, j)$ is $O(n)$.

For this claim, consider a pair $(i, j)$. During the rotation, the first intersection point moves along the boundary of disk $D_{i}$ and the last intersection point moves along the boundary of disk $D_{j}$. Therefore, the movement of $C_{2}$ is determined by these two intersection points. 
Clearly $C_{1}$ has at most $2(n-1) \mathrm{I} / \mathrm{O}$ events. For $C_{2}$, the trajectory of its center is a function graph which "behaves well" - Since it is a function on the radii of disks $D_{i}$ and $D_{j}$, and their center locations, it is not in a complicated form (and its degree is low enough) that there are only $O(n)$ events.

We compute all I/O events and sort them. At the beginning of the rotation of $C_{1}$ around $D$, we compute the number of input disks that are not fully covered, and set the variable counter to this number. Then we handle I/O events one by one and update the counter. If the counter becomes 0 , we return "yes".

In total, case (d) can be handled in $O\left(n^{3} \log n\right)$ time.

Lemma 7. Given a value $r>0$, we can decide in $O\left(n^{3} \log n\right)$ time whether there exists two disks with radius $r$ that cover a set of given disks in the plane.

For the optimization algorithm we use parametric search.

To use the parametric search technique, we will design a parallel version of the decision algorithm. Then the overall algorithm runs in time $O\left(p \cdot T_{p}+T_{p} \cdot T_{d} \log p\right)$, where $p$ denotes the number of processors, $T_{d}$ denotes the running time of a decision algorithm, and $T_{p}$ denotes the running time of the parallel decision algorithm using $p$ processors. We have a parallel decision algorithm where $p=O\left(n^{3}\right)$ processors and $T_{p}=O\left(\log ^{2} n\right)$ time for some constant $c>1$. Thus the overall algorithm runs in time $O\left(n^{3} \log ^{4} n\right)$ time.

Parallel decision algorithm. For case (a), we assign $O(n)$ processors to each candidate $D$. The 1-center disk covering for the disks in $\mathcal{D} \backslash D$ can be computed by a known parallel linear programming algorithm [11] in $O\left(\log ^{2} n\right)$ time with $O(n)$ processors. Hence, we can solve case (a) in $O\left(\log ^{2} n\right)$ time with $O\left(n^{2}\right)$ processor.

For case (b), we assign $O(n)$ processors to each pair $\left(D, D^{\prime}\right)$ of input disks. With a covering disk $C_{1}$ of radius $r$ determined by $D$ and $D^{\prime}$, we cover the input disks and compute the crescents of the input disks not covered by $C_{1}$ in a constant time. The 1-center disk covering for the crescents can be computed in $O\left(\log ^{2} n\right)$ time with $O(n)$ processors [11. Thus we can handle the case (b) in $O\left(\log ^{2} n\right)$ time with $O\left(n^{3}\right)$ processors, and moreover we can deal with case $(c)$ in a similar way.

For case (d), we first compute the union $U$ of all input disks in $O(\log n)$ time with $O\left(n^{3}\right)$ processors [15]. Next we assign $O\left(n^{2}\right)$ processors to each input disk $D$. We fix $D$. As $C_{1}$ rotates around $D$ while they keep touching as in Figure2(2), we need to figure out $O(n)$ pairs $(i, j)$ of input disks such that $D_{i}$ and $D_{j}$ are the first and the last ones intersected by $C_{1}$, respectively. Such $D_{i}$ and $D_{j}$ must be on the boundary of $U$, so it is sufficient to consider the disks whose arcs appear on the boundary of $U$. To get these pairs, we assign $O(n)$ processors to each disk on the union boundary in order to calculate two rotating angles of $C_{1}$ at which $C_{1}$ hits the disk at the first and the last in $O(1)$ time. We collect all these angles, sort them, and extract the pairs $(i, j)$ from the sorted list; all steps are easily done in $O(\log n)$ time using $O(n)$ processors.

For a fixed angle interval $I$ determined by some pair $(i, j)$, the set of input disks not covered by $C_{1}$ remains same, and we can also know which disks are those ones. Using $O(n)$ processors in $O(1)$ time for each input disk $D^{\prime}$ not covered by $C_{1}$, we compute the subintervals $J \subseteq I$ such that $C_{1} \cup C_{2}$ determined by $J$ contains $D^{\prime}$ at any angle in $J$. These subintervals are defined by I/O events we mentioned in the sequential decision algorithm, so there are $O(n)$ subintervals. Finally we test whether the intersection of the subintervals is empty or 
not. If it is not empty, then it means there is a rotation angle in $I$ at which all input disks not covered by $C_{1}$ get to be contained in $C_{1} \cup C_{2}$. Otherwise, no angles in $I$ guarantee the full coverage by $C_{1} \cup C_{2}$. This test can be done in bottom-up fashion in $O(\log n)$ time using $O(n)$ processors. After testing all pairs $(i, j)$, if there is a pair such that the intersection is not empty, then return "yes". This is done in $O(\log n)$ time with $O\left(n^{2}\right)$ processors for a fixed disk $d$ touching $C_{1}$ from inside. Summing up all things, we can solve case (d) in $O(\log n)$ time with $O\left(n^{3}\right)$ processors.

Theorem 8. Given a set of $n$ disks in the plane, we can find a pair of congruent disks with smallest radius whose union covers all of them in $O\left(n^{3} \log ^{4} n\right)$ time.

\subsubsection{Constant Factor Approximation.}

We apply the well known greedy $k$-center approximation algorithm by Gonzalez 10 to our general covering case. It works as follows: First pick an arbitrary point $c_{1}$ in the union $\bigcup \mathcal{D}$ of our input disks. For instance, we could choose $c_{1}$ to be the center of $D_{1}$. Then compute a point $c_{2} \in \bigcup \mathcal{D}$ that is farthest from $c_{1}$. This can be done in linear time by brute force. These two points are the centers of our two covering disks, and we choose their radius to be as small as possible, that is, the radius of the two covering disks is the maximum distance from any point in $\bigcup \mathcal{D}$ to its closest point in $\left\{c_{1}, c_{2}\right\}$. This algorithm is a 2-approximation algorithm, so we obtain the following result:

Theorem 9. We can compute in $O(n)$ time a 2-approximation for the general covering problem for a set $\mathcal{D}$ of $n$ disks.

\subsection{3 $(1+\epsilon)$-Approximation.}

Our $(1+\epsilon)$-approximation algorithm is an adaptation of an algorithm by Agarwal and Procopiuc [1]. We start by computing a 2-approximation for the general covering case in $O(n)$ time using our algorithm from Theorem 9. Let $C_{1}, C_{2}$ be the disks computed by this approximation algorithm and let $r$ be their radius. We consider a grid of size $\delta=\lambda \epsilon r$ over the plane, where $\lambda$ is a small enough constant. That is, we consider the points with coordinates $(i \delta, j \delta)$ for some integers $i, j$. Observe that there are only $O\left(1 / \epsilon^{2}\right)$ grid points in $C_{1} \cup C_{2}$. The center of each disk $D$ is moved to a nearby grid point. That is, a center $(x, y)$ is replaced by $(\delta\lceil x / \delta\rceil, \delta\lceil y / \delta\rceil)$. If two or more centers are moved to the the same grid point, we only keep the disk with the largest radius. All the centers are now grid points inside $C_{1} \cup C_{2}$, or at distance at most $\sqrt{2} \delta$ from the boundary of this union, so we are left with a set of $O\left(1 / \epsilon^{2}\right)$ disks. We now replace this new set of disks by grid points: each disk is replaced by the grid points which are closest to the boundary of this disk and lie inside this disk. In order to compute these points we consider each column of the grid separately: The intersection of each disk with this column is an interval, and we replace the interval by the lowest and the highest grid point lying inside this interval. Since the set of disks has size $O\left(1 / \epsilon^{2}\right)$ and the number of columns is $O(1 / \epsilon)$, it takes in total $O\left(1 / \epsilon^{3}\right)$ time. The set of grid points we obtain is denoted by $P_{g}$ and its size is $O\left(1 / \epsilon^{2}\right)$. We compute two smallest disks $E_{1}, E_{2}$ that cover $P_{g}$ in $O\left(\frac{1}{\epsilon^{2}} \log ^{2} \frac{1}{\epsilon} \log ^{2} \log \frac{1}{\epsilon}\right)$ time using the algorithm from Chan [4]. Choosing the constant $\lambda$ small enough and increasing the radii of $E_{1}, E_{2}$ by $2 \sqrt{2} \delta$, these disks are a $(1+\epsilon)$-approximation of the solution to our general disk cover problem. 
Theorem 10. Given a set $\mathcal{D}$ of $n$ disks in the plane, a $(1+\epsilon)$-approximation for $\mathcal{D}$ in the general covering case can be computed in $O\left(n+1 / \epsilon^{3}\right)$ time.

\subsection{The Restricted Case}

Observation 1 can be adapted to the restricted covering case.

Observation 11. Let $\ell$ be the bisector of an optimal solution $C_{1}$ and $C_{2}$. Then, $D \subset C_{i}$ for every $D \in \mathcal{D}$ whose center lies in the same side of $\ell$ as the center of $C_{i}$, for $i=\{1,2\}$.

Hence, the restricted covering problem can be solved in $O\left(n^{3}\right)$ time, since for a set of $n$ disks $\mathcal{D}$ the smallest disk covering all $D \in \mathcal{D}$ can be computed in $O(n)$ time [13, and there are $O\left(n^{2}\right)$ different bipartitions of the centers of the disks.

The algorithm from Section 2 can also be adapted to solve the restricted covering problem. We consider the decision problem, which can be formulated as follows: Given a set of $n$ disks $\mathcal{D}$ and a value $\delta$, we want to decide whether there exists two disks $C_{1}, C_{2}$ with radius $\delta$, such that each disk $D_{i} \in \mathcal{D}$ is covered by either $C_{1}$ or $C_{2}$. This implies that for each disk $D_{j} \in \mathcal{D}$ covered by $C_{i}$, the following holds: $d\left(c\left(D_{j}\right), c\left(C_{i}\right)\right)+r\left(D_{j}\right) \leq \delta$, for $i=\{1,2\}$. Let $r_{\max }$ be the maximum of radii of all disks in $\mathcal{D}$. It holds that $\delta \geq r_{\max }$, since if $\delta<r_{\text {max }}$ there clearly exists no two disks with radius $\delta$ which cover $\mathcal{D}$. We can formulated the problem in a different way.

Given a value $\delta$, do there exist two points, $p_{1}$ and $p_{2}$, in the plane such that $D^{*}(\delta) \cap\left\{p_{1}, p_{2}\right\} \neq \emptyset$ for every $D \in \mathcal{D}$, where $D^{*}(\delta)$ is a disk concentric to $D$ and whose radius is $\delta-r(D) \geq 0$.

Recall the definition of $\delta$-inflated disks from Section 2, Every disk $D \in \mathcal{D}$ was replaced by a disk concentric to $D$ and whose radius was $r(D)+\delta$. Here we actually need to replace each disk $D$ by a disk that is concentric to $D$ and has a radius $\delta-r(D)$. Since we know that $\delta \geq r_{\max }$, we add an initialization step, in which every disk $D$ is replaced by a disk concentric to $D$ and whose radius is $r_{\max }-r(D)$. Then we can use exactly the same algorithm in Section 2 in order to compute a solution for the restricted covering problem. Let $\delta^{*}$ be the solution value computed by this algorithm. Clearly the solution for the covering problem is then $\delta^{*}+r_{\max }$. We summarize this result in the following theorem.

Theorem 12. Given a set of $n$ disks $\mathcal{D}$ in the plane, we can compute two smallest congruent disks such that each disk in $\mathcal{D}$ is covered by one of the disks in $O\left(n^{2} \log ^{3} n\right)$ expected time or in $O\left(n^{2} \log ^{4} n \log \log n\right)$ worst-case time.

\subsubsection{Constant Factor Approximation.}

Let $C_{1}, C_{2}$ denote an optimal solution to the general case, and let $r_{g}$ be their radius. Then any solution to the restricted case is also a solution to the general case, so we have $r_{g}$ is at most the radius of the optimal solution to the restricted case. On the other hand, the inflated disks $C_{1}\left(2 r_{g}\right), C_{2}\left(2 r_{g}\right)$ form a solution to the restricted case, because any disk contained in $C_{1} \cup C_{2}$ should be contained in either $C_{1}\left(2 r_{g}\right)$ or $C_{2}\left(2 r_{g}\right)$. So we obtain a 6-approximation algorithm for the restricted case by first applying our 2-approximation algorithm for the general case (Theorem 9) and then multiplying by 3 the radius of the two output disks: 
Theorem 13. Given a set of $n$ disks $\mathcal{D}$ in the plane, we can compute in $O(n)$ time a 6 approximation to the restricted covering problem.

As in the general case, we will see below how to improve it to a linear time algorithm for any constant approximation factor larger than 1.

\subsection{2 $(1+\epsilon)$-Approximation.}

Recall Observation 11. Let $\ell$ be the bisector of an optimal solution. Then each disk $D \in \mathcal{D}$ is covered by the disk $C_{i}$ whose center lies in the same side of the center of $C_{i}, i \in\{1,2\}$. Hence, if we know the bisector, we know the bipartition of the disks. First, we show how to compute an optimal solution in $O(n \log n)$ time if the direction of the bisector is known. Later on we explain how this algorithm is used in order to obtain a $(1+\epsilon)$ approximation.

Fixed Orientation. W.l.o.g, assume that the bisector is vertical. After sorting the centers of all $D \in \mathcal{D}$ by their $x$-values, we sweep a vertical line $\ell$ from left to right, and maintain two sets $\mathcal{D}_{1}$ and $\mathcal{D}_{2}: \mathcal{D}_{1}$ contains all disks whose centers lie to the left of $\ell$ and $\mathcal{D}_{2}=\mathcal{D} \backslash \mathcal{D}_{1}$. Let $C_{1}$ be the smallest disk covering $\mathcal{D}_{1}$ and $C_{2}$ the smallest disk covering $\mathcal{D}_{2}$. While sweeping $\ell$ from left to right, the radius of $C_{1}$ is nondecreasing and the radius of $C_{2}$ nonincreasing and we want to compute $\min \max \left(r\left(C_{1}\right), r\left(C_{2}\right)\right)$. Hence, we can perform a binary search on the list of the centers of the disks in $\mathcal{D}$. Each step takes $O(n)$ time, thus we achieve a total running time of $O(n \log n)$.

Sampling. We use $2 \pi / \epsilon$ sample orientations chosen regularly over $2 \pi$, and compute for each orientation the solution in $O(n \log n)$ time. The approximation factor can be proven by showing that there is a sample orientation that makes angle at most $\epsilon$ with the optimal bisector. Without loss of generality, we assume that the bisector, denoted by $b$, of an optimal solution $C_{1}^{*}$ and $C_{2}^{*}$ is vertical as in Figure 3. Let $q$ denote the midpoint of the segment connecting $c\left(q_{1}\right)$ and $c\left(q_{2}\right)$.

Let $\ell$ be the line which passes through $q$ and makes angle with $b$ is at most $\epsilon$ in counterclockwise direction as in the figure. (For simplification we set the angle in the calculation to exactly $\epsilon$.) Let $p$ denote the intersection point of $\ell$ with the upper circular arc of $\partial C_{1}^{*}$, and let $p^{\prime}$ denote the point symmetric to $p$ along $b$. Clearly $p^{\prime}$ lies on the boundary of $C_{2}^{*}$. We will show that there exists two disks $C_{1}, C_{2}$ where $C_{1}$ covers all disks whose centers lie to the left of $\ell$ and $C_{2}$ covers all disks whose centers lie to the right of $\ell$ and $r\left(C_{1}\right)=r\left(C_{2}\right) \leq(1+\epsilon) r\left(C_{1}^{*}\right)$.

We will explain the construction of $C_{2}$ and prove that $C_{2}$ covers all disks whose centers lie to the right of $\ell . C_{1}$ can be constructed analogously. The center of $C_{2}$ is set to $c\left(C_{2}^{*}\right)$ and the radius is set to $\left|c\left(C_{2}^{*}\right) p\right| \leq\left|\overline{c\left(C_{2}^{*}\right) p^{\prime}}\right|+\left|\overline{p^{\prime} p}\right|$. It holds that $\left|\overline{c\left(C_{2}^{*}\right) p^{\prime}}\right|+\left|\overline{p^{\prime} p}\right| \leq r\left(C_{2}^{*}\right)+4 r\left(C_{2}^{*}\right) \sin \epsilon$, since $\left|\overline{p^{\prime} q}\right| \leq 2 r\left(C_{2}^{*}\right)$ and the distance of $p^{\prime}$ to $b$ is at most $2 r\left(C_{2}^{*}\right) \sin \epsilon$. Clearly $C_{2}$ covers all disks that were covered by $C_{2}^{*}$. In addition, it must cover all disks whose centers lie in the region of $C_{1}^{*}$ that is bounded by $\ell$ and $b$ and that has $q$ as its lowest point, depicted as the dark gray region in Figure 3. Note that the disks whose centers lie in this region are fully covered by $C_{1}^{*}$, but not necessarily by $C_{2}^{*}$.

It remains to prove that all disks having their center in the dark gray region are fully covered by $C_{2}$. Let $C^{\prime}$ be the disk symmetric to $C_{1}^{*}$ along $\ell$. Then all disks whose centers lie in the dark gray region are covered by $C_{1}^{*} \cap C^{\prime}$, because this region is symmetric along $\ell$ and 


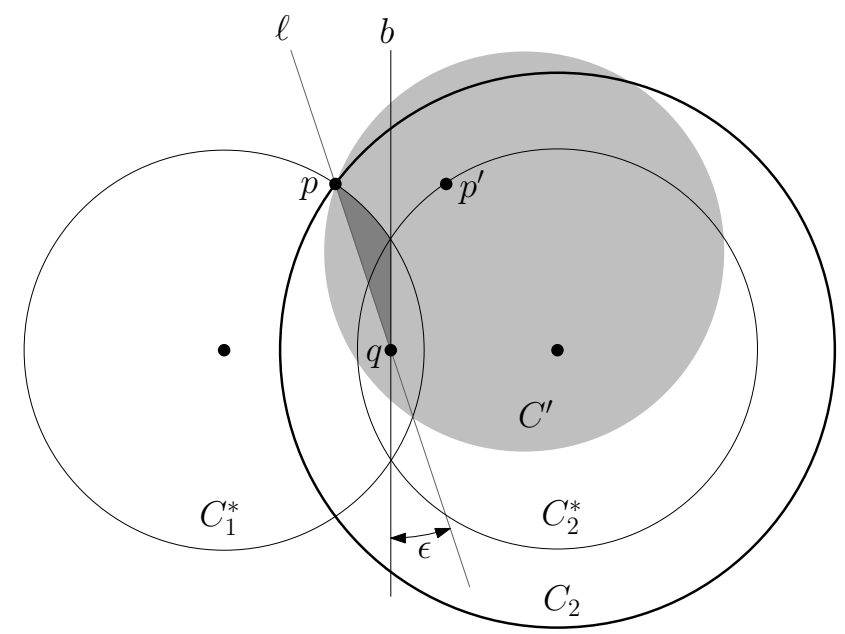

Figure 3: $r\left(C_{2}\right) \leq\left(1+\epsilon^{\prime}\right) r\left(C_{2}^{*}\right)$ for any $\epsilon^{\prime} \geq 4 \epsilon$.

they are fully covered by $C_{1}^{*}$. Since $C_{2}$ contains the intersection $C_{1}^{*} \cap C^{\prime}$, we conclude that all disks whose centers lie on the right side of $\ell$ are covered by $C_{2}$.

We can prove the analog for $C_{1}$. Hence,

$$
r\left(C_{1}\right)=r\left(C_{2}\right) \leq(1+4 \sin \epsilon) r\left(C_{1}^{*}\right) \leq\left(1+\epsilon^{\prime}\right) r\left(C_{1}^{*}\right)=\left(1+\epsilon^{\prime}\right) r\left(C_{2}^{*}\right)
$$

as $\sin \epsilon \leq \epsilon$ for $\epsilon \leq 1$ (can be shown by using the theory of Taylor series) and for any $\epsilon^{\prime} \geq 4 \epsilon$. Since any solution whose bisector is parallel to $\ell$ has a radius at most $r\left(C_{1}\right)$, this solution has radius at most $\left(1+\epsilon^{\prime}\right)$ times the optimal radius.

Theorem 14. For a given a set $\mathcal{D}$ of $n$ disks in the plane, a $(1+\epsilon)$ approximation for the restricted covering problem for $\mathcal{D}$ can be computed in $O((n / \epsilon) \log n)$ time.

The running time can be improved to $O\left(n+1 / \epsilon^{3} \log 1 / \epsilon\right)$ in the following way. We start with computing a 6-approximation in $O(n)$ time, using Theorem 13 . Let $C_{1}^{\prime}$ and $C_{2}^{\prime}$ be the resulting disks, and let $r^{\prime}$ be their radius. As in the proof of Theorem 10, we round the centers of all input disks $D \in \mathcal{D}$ to grid points inside $C_{1}^{\prime} \cup C_{2}^{\prime}$, with a grid size $\delta^{\prime}=\lambda^{\prime} \epsilon r^{\prime}$, for some small enough constant $\lambda^{\prime}$. Then we apply our FPTAS from Theorem 14 to this set of rounded disks and inflate the resulting disks by a factor of $\sqrt{2} \delta$. These disks are a $(1+\epsilon)$-approximation for the optimal solution. As there are only $O\left(1 / \epsilon^{2}\right)$ rounded disks, this can be done in $O\left(\left(1 / \epsilon^{3}\right) \log 1 / \epsilon\right)$ time.

Theorem 15. For a given a set $\mathcal{D}$ of $n$ disks in the plane, a $(1+\epsilon)$ approximation for the restricted covering problem for $\mathcal{D}$ can be computed in $O\left(n+\left(1 / \epsilon^{3}\right) \log 1 / \epsilon\right)$ time.

\section{Acknowledgment}

Work by Ahn was supported by the National Research Foundation of Korea Grant funded by the Korean Government (MEST) (NRF-2010-0009857). Work by Schlipf was supported by the German Science Foundation (DFG) within the research training group 'Methods for Discrete Structures'(GRK 1408). Work by Shin was supported by the National Research Foundation of Korea Grant funded by the Korean Government (MEST) (NRF-2011-0002827). 


\section{References}

[1] P. Agarwal and C. Procopiuc. Exact and approximation algorithms for clustering. Algorithmica, 33(2):201-226, 2002.

[2] P. K. Agarwal, M. Sharir, and S. Toledo. Applications of parametric searching in geometric optimization. J. Algorithms, 17:292-318, 1994.

[3] T. M. Chan. Deterministic algorithms for 2-d convex programming and 3-d online linear programming. J. Algorithms, 27(1):147-166, 1998.

[4] T. M. Chan. More planar two-center algorithms. Comput. Geom. Theory Appl., 13:189-198, 1999.

[5] K. L. Clarkson. Las Vegas algorithms for linear and integer programming when the dimension is small. J. ACM, 42:488-499, 1995.

[6] M. de Berg, O. Cheong, M. van Kreveld, and M. Overmars. Computational Geometry Algorithms and Applications. Springer-Verlag, 3 edition, 2008.

[7] Z. Drezner. The planar two-center and two-median problems. Transportation Science, 18:351-361, 1984.

[8] D. Eppstein. Faster construction of planar two-centers. In Proc. of SODA'97, pages 131-138, 1997.

[9] K. Fischer and B. Gartner. The smallest enclosing ball of balls: combinatorial structure and algorithms. In Proc. of SoCG'03, pages 292-301, 2003.

[10] T. Gonzalez. Clustering to minimize the maximum intercluster distance. Theor. Comput. Sci., 38:293-306, 1985.

[11] M. T. Goodrich. Fixed-dimensional parallel linear programming via relative $\epsilon$-approximations. In Proc. of SODA'96, pages 132-141, 1996.

[12] M. Löffler and M. van Kreveld. Largest bounding box, smallest diameter, and related problems on imprecise points. Comput. Geom. Theory Appl., 43:419-433, 2010.

[13] N. Megiddo. On the ball spanned by balls. Discr. Comput. Geom., 4:605-610, 1989.

[14] K. Mulmuley. Computational Geometry - An Introduction Through Randomized Algorithms. Prentice Hall, 1994.

[15] T. Papatheodoro and P. Spirakis. $O(\log n)$ parallel time intersection and union algorithms for a set of planar discs. Technical report, Courant Institute of Mathematical Sciences, New York University, 1987.

[16] M. Sharir and E. Welzl. A combinatorial bound for linear programming and related problems. In Proc. of STACS'92, pages 567-579, 1992.

[17] C.-S. Shin, J.-H. Kim, S. K. Kim, and K.-Y. Chwa. Two-center problems for a convex polygon. In Proc. of ESA'98, pages 199-210, 1998. 